\title{
ERP et profil d'activités du contrôleur de gestion : une évolution par paliers ?
}

\section{ERP and management controller activities profile: a stepwise evolution?}

Anass Mawadia est doctorant en sciences de gestion à l'Université de Montpellier. Chef de projet informatique dans des multinationales pendant une dizaine d'années, ses recherches portent sur l'implémentation de progiciels de gestion intégrée, vecteurs d'innovations managériales.

Adresse : MRM, Université de Montpellier, Place Eugène Bataillon - Bât 19, 34095 Montpellier

Téléphone : 0761498632

Courriel : a.mawadia@gmail.com

Philippe Chapellier est Professeur des Universités à Polytech Montpellier. Il est membre du laboratoire MRM et du Labex «Entreprendre ». Ses travaux portent sur les rôles des outils de contrôle de gestion, notamment en PME, et sur les transferts de connaissances entre professionnels comptables et dirigeants de PME.

Adresse : Polytech, Département Informatique et Gestion, Place Eugène Bataillon, 34095 Montpellier

Téléphone : 0670280146

Courriel : Philippe.Chapellier@umontpellier.fr

Ariel Eggrickx est Maître de Conférences, Habilitée à Diriger des Recherches en Sciences de Gestion, à l'Université de Montpellier. Membre du laboratoire «Montpellier Recherche Management », ses travaux de recherche portent sur le sens générique du contrôle et ses ressorts fondamentaux, la régulation de l'action collective qui repose sur des structures à la fois formelles et informelles, dans le champ des PME et organisations publiques (Hôpitaux, Universités).

Adresse : IUT de Montpellier, Département Informatique, 99, avenue d'Occitanie 34296 Montpellier Cedex 5

Téléphone : 06.87.55.65.42

Courriel : ariel.eggrickx@univ-montp2.fr

\footnotetext{
${ }^{1}$ Ce papier est une version modifiée en profondeur d'un article intitulé «ERP et profil d'activités du contrôleur

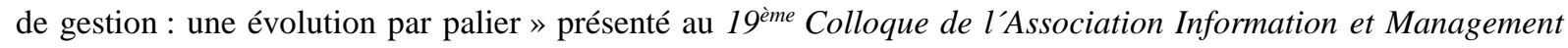
(AIM), Aix-en-Provence, 19-21 mai 2014
} 


\title{
ERP et profil d'activités du contrôleur de gestion : une évolution par paliers?
}

\author{
ERP and management controller activities profile: a stepwise evolution?
}

Résumé : Cet article étudie l'évolution du profil d'activités des contrôleurs de gestion après la mise en place d'un ERP (Enterprise Resource planning). Il s'appuie sur le modèle de Besson (1999) qui dresse les profils d'activités des contrôleurs de gestion avant et après la mise en place d'un ERP. Une enquête a été réalisée par questionnaire, puis par entretiens directs, dans une entreprise multinationale ayant mis en place l'ERP JD Edwards. Si l'ERP participe à l'évolution du profil d'activités du contrôleur de gestion, la mutation du métier vers un rôle de support au pilotage suppose une double condition : la mise en place d'outils de business intelligence et une volonté forte de la direction.

Mots clés : ERP, contrôleur de gestion, profil d'activités, business intelligence

Abstract: This paper studies the evolution of the management controller activities profile after an ERP (Enterprise Resource planning) implementation. The study is based on Besson model (1999) drawing up the management controller activity profile before and after ERP deployment. A survey was conducted using questionnaire, and then directs interviews in a multinational that installed the ERP JD Edwards. The results show that the ERP contributes to the evolution of the management controller activity profile. But the job mutation towards a steering support role implies double conditions: the implementation of business intelligence tools and a strong willingness from the top management.

Key words: ERP, management controller, activities profile, business intelligence

\section{Introduction}

Durant ces quinze dernières années, de nombreuses entreprises ont adopté des systèmes ERP (Enterprise Resource Planning) afin de centraliser l'ensemble de leurs activités dans un seul et unique système d'information.

Reix et al. (2011) définissent le système d'information comme "un ensemble organisé de ressources : matériel, logiciel, personnel, données, procédures permettant d'acquérir, traiter, stocker, communiquer des informations (sous forme de données, textes, images, sons, etc...) dans et entre des organisations » (p.4) et un ERP comme « une application informatique paramétrable, modulaire et intégrée, qui vise à fédérer et à optimiser les processus de gestion de l'entreprise en proposant un référentiel unique et en s'appuyant sur des règles de gestion standards » (p.97).

L'implantation d'ERP provoque de forts changements organisationnels (Rowe, 1999 ; Reix, 1999), et devrait contribuer à faire évoluer le profil d'activités du contrôleur de gestion (Besson, 1999). Cependant, les contrôleurs de gestion évoquent, suite à l'adoption d'un ERP, des charges de travail encore fastidieuses pour le reporting, et les recherches aboutissent à des résultats contradictoires quant aux impacts sur l'activité des contrôleurs de gestion. Certaines constatent que l'adoption d'un système ERP a des impacts relativement limités sur le profil d'activités des contrôleurs de gestion (Granlund et Malmi, 2002 ; Meyssonnier et Pourtier, 2006 ; O’Mahony et Doran, 2008). D'autres montrent en revanche que la mise en place d'un ERP génère des changements significatifs : un rôle plus important au sein de l'organisation (Cross, 2001), ou un élargissement à des rôles de conseiller et de consultant interne auprès d'autres managers (Scapens et Jazayeri, 2003 ; Grabski et al, 2009). Besson (1999) dresse un portrait avant / après (qu'il nomme «hier»/ «demain ») du profil d'activités des contrôleurs de gestion, et anticipe une évolution importante des rôles et fonctions des contrôleurs de gestion suite à l'implantation d'un ERP. La revue de littérature aboutissant à des conclusions contradictoires, l'objectif de la recherche consiste à déterminer dans quelle mesure et sous quelles conditions, le profil d'activités des contrôleurs de gestion évolue suite à l'implantation d'un ERP.

Dans une première partie, nous présentons les propositions de recherche à partir d'une revue de littérature au regard des modèles « hier » et « demain » proposés par Besson (1999). La seconde partie présente les différentes phases de l'enquête: l'administration d'un questionnaire auprès des 
contrôleurs de gestion d'une entreprise multinationale, puis la conduite d'entretiens semi-directifs auprès de six d'entre eux. La troisième partie expose les principaux résultats : 1 . Une part encore significative de tâches dites traditionnelles (fiabilisation et consolidation des données), 2. Une plus forte implication dans la conception et gestion des SIG, 3. Une participation plus forte au pilotage possible sous deux conditions : mise en place d'un outil de business intelligence (BI), forte volonté de la direction. Dans une quatrième partie, la discussion des résultats montre que l'évolution de la fonction de contrôleur de gestion vers des activités décisionnelles et stratégiques semble suivre un modèle par paliers.

\section{Le modèle du contrôleur de gestion de « demain » : mythe ou réalité ?}

La revue de littérature sur l'évolution du profil d'activités des contrôleurs de gestion suite à la mise en place d'un ERP (1.1) conduit à formuler les propositions de recherche (1.2).

\subsection{Des recherches qui aboutissent à des résultats plutôt contradictoires}

Plusieurs travaux se sont récemment centrés sur la fonction de contrôleur de gestion. Lambert et Sponem (2009) étudient la place et le rôle de cette fonction dans l'organisation et mettent en évidence quatre idéaux types : les fonctions contrôle de gestion discrète, garde-fou, partenaire et omnipotente. Bollecker (2011) se focalise sur la socialisation des contrôleurs de gestion et identifie trois profils différents : les légitimés, les marginalisés et les intégrés. Ces typologies se centrent sur la fonction de contrôleur de gestion et pas sur leur profil d'activités. Partant de l'hypothèse d'un impact des ERP, Besson (1999) réalise une analyse du profil d'activités d'environ cinquante contrôleurs de gestion du groupe Autoroutes du Sud de la France (ASF) exerçant dans un environnement relativement stable et peu concurrentiel. Il identifie douze activités (P1 à P12) qu'il regroupe en quatre volets : participer au reporting (P1 à $\mathrm{P} 3)$, participer à la planification (P4 à $\mathrm{P} 6)$, participer au pilotage ( $\mathrm{P} 7$ à $\mathrm{P} 9)$ et gérer le système d'information de gestion (P10 à P12). Le premier profil (hier) rend compte de la perception par les contrôleurs de gestion de ces douze activités avant l'implantation de l'ERP. Le second (demain) montre l'évolution attendue par les contrôleurs de ces douze activités après l'implantation de l'ERP.

Figure 1 : Le profil d'activités du contrôleur de gestion avant (hier) et après (demain) la mise en place d'un ERP (Besson, 1999)

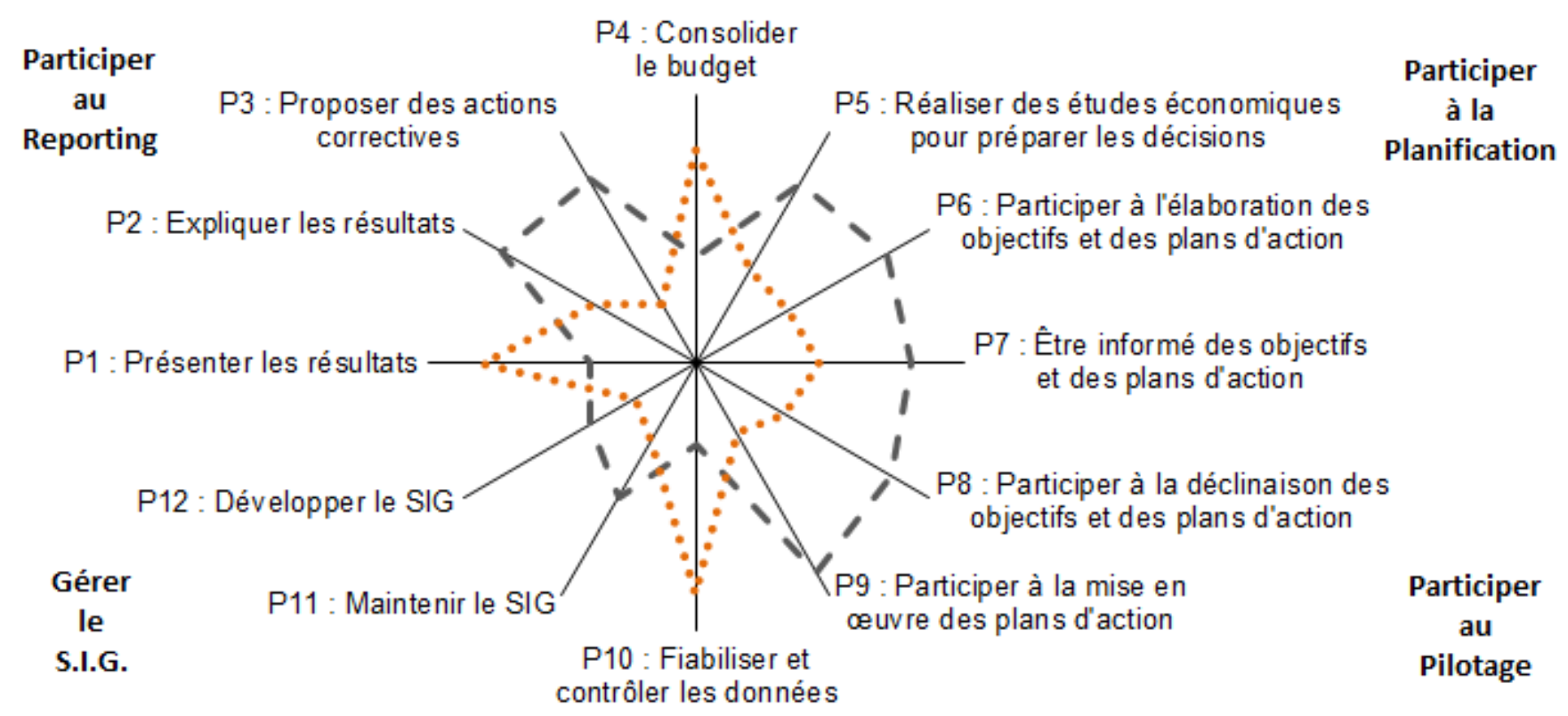

- Profil du contrôleur de gestion "demain"

.....Profil du contrôleur de gestion "hier" 
Besson (1999, p.5) explique que «le métier de contrôleurs de gestion s'enrichit significativement avec l'implantation de l'ERP. Le métier actuel est focalisé sur les tâches ingrates, notamment le recueil, la fiabilisation et la consolidation des données, alors que le métier de demain se structurera autour des tâches nobles de modélisation et d'interprétation des données de gestion ». Il affirme que : «Toute chose égale par ailleurs, un progiciel ERP est à la chaîne comptable-financière ce que le robot a été pour les chaînes d'assemblage. Comme l'ouvrier spécialisé de l'industrie automobile, le contrôleur de gestion assiste impuissant à la disparition de son ancien métier ». La grille utilisée par Besson (1999) n'a, à notre connaissance, pas été mobilisée par d'autres chercheurs. Mais plusieurs recherches ont été réalisées sur le thème de l'impact de l'adoption d'un ERP sur l'activité des contrôleurs de gestion. Elles aboutissent à des résultats contradictoires.

Certaines confirment, de façon plus ou moins partielle, les évolutions attendues selon le modèle de Besson (1999). Cross (2001) souligne par exemple que les systèmes ERP élargissent le champ de travail des contrôleurs de gestion et génèrent une évolution du rôle traditionnel de «comptables de coûts » vers un rôle plus stratégique. Scapens et Jazayeri (2003) estiment que l'adoption d'un système ERP fait évoluer le rôle des contrôleurs de gestion car les directeurs ont besoin de leur aide pour comprendre l'information fournie par l'ERP. Les ERP élargiraient le rôle des contrôleurs de gestion et ceux-ci deviendraient des conseillers internes auprès d'autres managers. Chtioui et Bennani (2009, p.13) expliquent que la mise en place d'un ERP « ouvre de nouvelles perspectives pour une meilleure valorisation du travail du contrôleur de gestion grâce à une focalisation accrue sur l'analyse et le conseil aux dépens des tâches routinières plus classiques ». Grabski et al. (2009) constatent que l'installation réussie de l'ERP amène des changements significatifs dans les missions du contrôleur de gestion : ce dernier devient plus étroitement impliqué dans la prise de décision et effectue d'autres tâches à forte valeur ajoutée.

Mais d'autres recherches ne constatent pas les évolutions attendues selon le modèle de Besson (1999). Granlund et Malmi (2002) précisent que, contrairement aux attentes, l'adoption d'un ERP permet certes de réduire la charge de travail des contrôleurs de gestion, mais que ceux-ci ne deviennent pas pour autant des conseillers auprès des managers. Dans la même optique, O'Mahony et Doran (2008, p.113) admettent un impact limité de l'ERP sur le rôle du contrôleur de gestion et affirment que "l'implémentation des systèmes ERP n'a pas changé la responsabilité ultime des contrôleurs de gestion qui est la préparation et la validation des chiffres mensuels ». Meyssonnier et Pourtier (2006, p.61) relativisent également l'impact de l'ERP sur les fonctions des contrôleurs de gestion : "nous n'avons pas pu constater la dissociation fonctionnelle annoncée en matière de responsabilités des contrôleurs de gestion. L'évolution ne se traduit pas toujours par une émancipation des contraintes de collecte et de mise en forme des informations et ne se fait que peu au profit de tâches plus stratégiques liées au conseil des managers. Cela peut même être l'inverse! ». Ils expliquent que les contrôleurs de gestion se trouvent souvent «embourbés »dans la collecte et la vérification de la qualité des données, ce qui les conduit à proposer «une représentation des évolutions possibles de la fonction contrôleur de gestion en environnement ERP beaucoup plus limitée que beaucoup d'auteurs $»(\mathrm{p} .62)$.

Ces résultats contradictoires peuvent être liés à diverses raisons : l'implémentation plus ou moins récente de l'ERP au moment de la recherche, son périmètre, l'implication des utilisateurs dans le projet ERP ou le degré de succès de l'implémentation (Grabski et al., 2009, 2011 ; Gullkvist, 2013), les caractéristiques de l'organisation - âge, taille, structure, secteur d'activité - (Gullkvist, 2013), ou encore le positionnement du contrôleur de gestion, variable selon les attentes de la direction générale (Chtioui et Bennani, 2009). Ces résultats non définitifs appellent de nouvelles recherches afin de déterminer dans quelle mesure et sous quelles conditions le profil d'activités du contrôleur de gestion évolue dans les organisations suite à l'implantation d'un ERP.

\subsection{Les propositions de recherche}

Pour tenter de contribuer au débat, nous formulons douze propositions de recherche liées aux douze activités du métier de contrôleur de gestion retenues par Besson (1999) (voir figure 1). 


\section{Volet 1 : Participer au reporting}

Selon Besson (1999), la mise en place d'un ERP devrait conduire le contrôleur de gestion à passer moins de temps à présenter les résultats (information disponible et facile à obtenir en temps réel) et plus de temps à les expliquer et à prendre des mesures correctives. Le rôle du contrôleur serait ainsi davantage centré sur la création de la valeur. La majorité des recherches confirme que le contrôleur de gestion passe moins de temps à la présentation des résultats suite à la mise en place d'un ERP (Granlund et Malmi, 2002 ; Scapens et al., 1998 ; Scapens et Jazeyri, 2003 ; Meyssonnier et Pourtier, 2006 ; O’Mahony et Doran, 2008). Mais seuls O’Mahony et Doran (2008) constatent une plus forte participation du contrôleur de gestion à l'explication des résultats et à la proposition de mesures correctives. Les décalages constatés entre les résultats de ces recherches nous conduisent à vérifier les propositions suivantes :

P1: La mise en place d'un ERP permet au contrôleur de gestion de consacrer moins de temps à la présentation des résultats

$P 2$ : La mise en place d'un ERP permet au contrôleur de gestion de participer plus intensément à l'explication des résultats

P3 : La mise en place d'un ERP permet au contrôleur de gestion de participer plus intensément à la proposition de mesures correctives.

\section{- Volet 2 : Participer à la planification}

Selon Besson (1999), la mise en place d'un ERP devrait conduire le contrôleur de gestion à passer moins de temps à la consolidation des budgets, et plus de temps d'une part, à la réalisation d'études économiques pour préparer les décisions et d'autre part, à l'élaboration des objectifs et des plans d'action. Granlund et Malmi (2002) et Grabski et al. (2009), en accord avec Besson, notent que l'ERP facilite le travail analytique. Coat et Favier (1999) qualifient l'ERP d'outil de planification et de suivi. Scapens et Jazeyri (2003) observent une participation accrue des contrôleurs de gestion à l'élaboration des objectifs et des plans d'action, variable selon l'expérience du système et le degré d'intégration de l'ERP. Chtioui et Bennani (2009) constatent une focalisation accrue sur l'analyse. D'autres auteurs ne sont en revanche que partiellement en accord avec Besson. Meyssonnier et Pourtier (2006) notent par exemple que le temps consacré au contrôle des états transmis et à leur mise en forme reste important. Ces résultats divergents nous conduisent à vérifier les propositions suivantes :

P4 : La mise en place d'un ERP permet au contrôleur de gestion de consacrer moins de temps à la consolidation des budgets.

P5 : La mise en place d'un ERP permet au contrôleur de gestion de participer davantage à la réalisation d'études économiques pour préparer les décisions.

P6 : La mise en place d'un ERP permet au contrôleur de gestion de participer davantage à l'élaboration des objectifs et plans d'action.

\section{- Volet 3 : Participer au pilotage}

Selon Besson (1999), les contrôleurs de gestion pourraient, grâce à l'ERP, être mieux informés sur les objectifs et plans d'action et participer davantage à leur déclinaison et à leur mise en œuvre. D'autres chercheurs confirment et constatent que, suite à l'implantation d'un ERP, les directeurs s'appuient davantage sur les contrôleurs de gestion pour comprendre et utiliser l'information disponible (Scapens et Jazeyri, 2003 ; Sangster et al., 2009 ; Grabski et al., 2011). Chtioui et Bennani (2009) concluent que l'ERP ouvre de nouvelles perspectives pour le contrôleur de gestion, avec une focalisation accrue sur le rôle d'analyse et de conseil. Ces auteurs expliquent ainsi que le contrôleur de gestion évolue d'un rôle de «comptable des coûts » vers un rôle plus stratégique (Cross, 2001), d'un rôle « d'enregistreur de chiffres » vers un rôle de «partenaire de gestion » (Caglio, 2003). Mais d'autres auteurs ne notent aucune participation plus intense du contrôleur de gestion au pilotage suite à l'implantation d'un ERP. Deux raisons principales sont avancées : des contrôleurs de gestion « embourbés » dans la collecte et vérification des données (Meyssonnier et Pourtier, 2006) et des objectifs et responsabilités se limitant à « fournir l'information »(O'Mahony et Doran, 2008 ; Boitier, 2008). Ces résultats contradictoires nous conduisent à vérifier les propositions suivantes : 
P7 : La mise en place d'un ERP permet au contrôleur de gestion d'être mieux informé des objectifs et des plans d'action.

P8 : La mise en place d'un ERP permet au contrôleur de gestion de participer davantage à la déclinaison des objectifs et des plans d'action.

P9 : La mise en place d'un ERP permet au contrôleur de gestion de participer davantage à la mise en cuvre des objectifs et des plans d'action.

\section{- Volet 4 : Gérer le système d'information de gestion}

Selon Besson (1999), la mise en place d'un ERP devrait permettre aux contrôleurs de gestion de consacrer moins de temps au contrôle et à la fiabilisation des données et de s'intéresser plus intensément au maintien et au développement du système d'information de gestion. Plusieurs auteurs confirment une activité du contrôleur plus orientée vers la conception (participation au cahier des charges, rédaction des procédures, paramétrages...) et la maintenance du système d'information de gestion (Burns et Scapens, 2000 ; Cross, 2001 ; Meyssonnier et Pourtier, 2006 ; Chtioui et Bennani, 2009 ; Grabski et al., 2009), ou vers des missions telles que la gestion des innovations technologiques (Caglio, 2003), l'audit, la maintenance, et la vérification de l'information contenue dans le système (Azan et Bollecker, 2011). Ces résultats, plutôt convergents, conduisent à formuler les propositions suivantes :

P10 : La mise en place d'un ERP permet au contrôleur de gestion de consacrer moins de temps au contrôle et à la fiabilisation des données.

P11 : La mise en place d'un ERP permet au contrôleur de gestion de s'intéresser plus intensément au maintien du système d'information de gestion.

P12 : La mise en place d'un ERP permet au contrôleur de gestion de s'intéresser plus intensément au développement du système d'information de gestion.

\section{Un pluralisme de méthodes pour observer, comprendre et interpréter}

Une étude a été menée au sein d'une multinationale de services (2.1) en deux phases : une première avec l'administration d'un questionnaire auprès des contrôleurs de gestion de l'entreprise, une seconde avec la réalisation d'entretiens semi-directifs (2.2).

\subsection{Une recherche réalisée dans une multinationale de services}

Notre étude a été réalisée entre 2010 et 2011 dans une multinationale de services (que nous nommerons Alpha) composée de 300 filiales réparties dans 120 pays sur les cinq continents et disposant d'un effectif de 59000 salariés. Cette organisation a lourdement investi en 2007 dans la mise en place de l'ERP JD Edwards (JDE) afin que toutes ses filiales utilisent un seul et même système d'information capable de standardiser les plates-formes d'information, d'unifier les méthodes de travail, et de fluidifier la remontée et la consolidation des informations.

Chez Alpha, le contrôle de gestion est organisé en deux niveaux : dans les filiales, un suivi opérationnel des activités et un reporting au groupe; au siège, un suivi et une consolidation des données.

Les contrôleurs de gestion du groupe Alpha ont participé à la mise en place du projet ERP tout au long de son cycle de vie: participation aux activités d'analyse des besoins, revue des processus métiers avec les managers, conception de l'outil avec la direction informatique, paramétrages et tests de l'ERP, mise à jour des données ou règles de gestion dans l'outil. Ils sont garants de l'adéquation des règles de gestion et des fichiers de reporting paramétrés dans l'ERP. Ils ont également joué un rôle de maîtrise d'ouvrage, d'intermédiaire/clarificateur entre les équipes informatiques et les managers utilisateurs des données générées par l'ERP.

La mise en place de l'ERP a suivi les étapes suivantes :

1- Session de pré-lancement (Pré-kick off) : Identification des besoins et revue de processus ;

2- Session de lancement (Kick off) : Rédaction du cahier des charges et planification ;

3- Session de test : recette et validation des tests ;

4- Session de démarrage (Go live) : Formation et mise en production ;

5- Session de clôture : Clôture du projet et résolution des problèmes en suspens. 
Les contrôleurs de gestion des filiales ont été impliqués dans chacune de ces étapes et dans les différentes demandes d'améliorations après la mise en place de l'ERP. Des sessions de formation ont par ailleurs été organisées pour les contrôleurs de gestion et les directeurs financiers.

La mise en place de l'ERP a entrainé des réorganisations dans les services financiers des filiales avec notamment la création de services de comptabilité partagée (Shared Service Centers) par pays.

\subsection{Une recherche en deux phases : questionnaires et entretiens}

Notre méthodologie de recherche est fondée sur une démarche d'étude de cas (Wacheux, 1996 ; Yin, 2008) et se caractérise par un pluralisme méthodologique (Woodside, 2010) visant à multiplier les sources de données (questionnaires et entretiens). Les postures épistémologiques positiviste et interprétativiste ont ainsi été associées. Le travail de terrain s'est déroulé en deux phases.

La première phase, menée trois ans après la fin de l'implémentation de l'ERP, est une analyse descriptive du profil d'activités des contrôleurs de gestion utilisant l'ERP «JD Edwards » dans l'entreprise Alpha. Son objectif est de confirmer (ou non) que ce profil d'activités est de type « demain » (selon le modèle proposé par Besson, 1999) suite à la mise en place de l'ERP.

Le questionnaire permet, pour chacune des douze activités identifiées par Besson, de recueillir la perception des contrôleurs de gestion sur une échelle de 1 à 5 . Les items utilisés pour procéder à cette mesure sont ceux utilisés par Besson dans son étude de 1999. Le questionnaire a été envoyé aux 108 contrôleurs de gestion utilisant cet ERP depuis au moins trois ans des 65 filiales du groupe Alpha situées dans dix pays différents (France, Royaume-Uni, Etats-Unis, Japon, Russie et Australie...). Les résultats obtenus sont présentés dans le tableau 1.

Tableau 1 : Résultats obtenus pour chacune des 12 propositions via l'analyse quantitative

\begin{tabular}{|c|c|c|c|c|c|}
\hline Propositions & N & Minimum & Maximum & Moyenne & Ecart type \\
\hline P1 & 30 & 1 & 5 & 4,07 & 3,258 \\
P2 & 30 & 1 & 5 & 3,87 & 1,279 \\
P3 & 30 & 1 & 5 & 2,00 & 1,174 \\
P4 & 30 & 1 & 5 & 2,67 & 1,363 \\
P5 & 30 & 1 & 5 & 2,47 & 1,213 \\
P6 & 30 & 1 & 5 & 2,40 & 1,332 \\
P7 & 30 & 1 & 5,40 & 1,102 \\
P8 & 30 & 1 & 5,00 & 1,102 \\
P9 & 30 & 1 & 5 & 3,60 & 1,486 \\
P10 & 30 & 1 & 5 & 3,00 & 1,102 \\
P11 & 30 & 1 & 4 & 2,47 & 1,114 \\
P12 & 30 & 1 & 5 & 0,973 \\
\hline
\end{tabular}

Nous avons obtenu 30 questionnaires dûment remplis, soit un taux de réponse de $28 \%$. Le tableau 2 présente les caractéristiques des 30 contrôleurs de gestion ayant répondu au questionnaire.

Tableau 2 : Caractéristiques des 30 contrôleurs de gestion ayant répondu au questionnaire

\begin{tabular}{|c|c|c|c|c|c|c|}
\hline Répondant & Pays & Sexe & Profil & $\begin{array}{c}\text { Année de présence dans } \\
\text { l'entreprise }\end{array}$ & $\begin{array}{c}\text { Expérience avec JD } \\
\text { Edwards (an) }\end{array}$ & Expérience avec un autre ERP \\
\hline 1 & Russie & Femme & CDG Filiale & 12 & 3 & 1S (ERP Local) \\
\hline 2 & Australie & Homme & CDG Filiale & 8 & 5 & Sage \\
\hline 3 & Angleterre & Homme & CDG filiale & 3 & 3 & Sage, SAP \\
\hline 4 & Irlande & Homme & CDG filiale & 3 & 3 & Exact \\
\hline 5 & Inde & Homme & CDG Filiale & 8 & 4 & Oracle \\
\hline 6 & Cote d'ivoire & Homme & CDG filiale & 10 & 5 & Sage \\
\hline 7 & Malaisie & Femme & CDG filiale & 2 & 3 & SAP \\
\hline 8 & Turquie & Femme & CDG filiale & 10 & 4 & SAP \\
\hline 9 & Italie & Homme & CDG Filiale & 4 & 3 & People soft \\
\hline 10 & Espagne & Homme & CDG Filiale & 3 & 4 & non \\
\hline 11 & France & Femme & CDG Filiale & 5 & 3 & non \\
\hline
\end{tabular}




\begin{tabular}{|c|c|c|c|c|c|c|}
\hline 12 & Japon & Homme & CDG filiale & 10 & 4 & SAP \\
\hline 13 & Slovénie & Femme & CDG filiale & 5 & 2 & Axapta \\
\hline 14 & Brésil & Homme & CDG filiale & 5 & 3 & Oracle \\
\hline 15 & USA & Homme & CDG Filiale & 5 & 3 & Oracle \\
\hline 16 & Ukraine & Femme & CDG Filiale & 5 & 3 & 1S (ERP Local) \\
\hline 17 & Russie & Femme & CDG activité & 10 & 3 & SAP Local) \\
\hline 18 & Dubai & Homme & CDG activité & 7 & 5 & SAP \\
\hline 19 & Hongrie & Homme & CDG activité & 5 & 5 & Axapta \\
\hline 20 & Angleterre & Femme & CDG activité & 2 & 3 & Sage \\
\hline 21 & Inde & Homme & CDG activité & 6 & 4 & 1S (ERP local) \\
\hline 22 & France & Femme & CDG activité & 6 & 5 & non \\
\hline 23 & Kazakhstan & Femme & CDG activité & 2 & 2 & non \\
\hline 24 & Turquie & Homme & CDG activité & $?$ & 2 & non \\
\hline 25 & Italie & Femme & CDG activité & 2 & 3 & Sage, people soft \\
\hline 26 & Espagne & Homme & CDG activité & 6 & 4 & Sage \\
\hline 27 & France & Homme & CDG activité & 6 & 4 & SAP \\
\hline 28 & France & Homme & CDG activité & 4 & 3 & Oracle \\
\hline 29 & Brésil & Homme & CDG activité & 2 & 3 & 5 \\
\hline
\end{tabular}

La seconde phase de l'étude, menée l'année suivante, est axée sur la compréhension et l'interprétation des causes de l'évolution ou non vers le profil d'activités de type «demain ». Plusieurs méthodes étaient utilisables pour valider et comprendre les résultats de l'étude quantitative. La technique des entretiens individuels en face-à-face nous est apparue la mieux adaptée, car elle permet d'accéder aux faits mais aussi aux représentations et aux interprétations des situations vécues et connues par les individus (Wacheux, 1996). Ont été sollicités pour un entretien le responsable du service contrôle de gestion «groupe » situé en France, et les 18 contrôleurs de gestion des filiales françaises et européennes ayant répondu aux questionnaires.

Six entretiens ont pu être réalisés (tableau 3) à partir d'un guide d'entretien : un avec le responsable du service contrôle de gestion «groupe », et cinq avec les contrôleurs de gestion filiales et activités qui ont accepté de nous recevoir (répondants 3, 11, 22, 27 et 28). L'entretien le plus court a duré 45 minutes et le plus long $1 \mathrm{~h} 10$.

Tableau 3 : Caractéristiques des 6 contrôleurs interviewés

\begin{tabular}{|l|c|c|c|c|c|c|}
\hline \multicolumn{1}{|c|}{ Personnes interviewées } & Sexe & $\begin{array}{l}\text { Année de } \\
\text { présence dans } \\
\text { l'entreprise }\end{array}$ & $\begin{array}{l}\text { Expérience } \\
\text { avec JD } \\
\text { Edwards (an) }\end{array}$ & $\begin{array}{l}\text { Expérience } \\
\text { avec un } \\
\text { autre ERP }\end{array}$ & Unité & Durée \\
\hline $\begin{array}{l}\text { Responsable Service contrôle } \\
\text { de gestion «groupe » }\end{array}$ & Homme & 6 & 5 & SAP & Siège & $\begin{array}{c}70 \\
\text { minutes }\end{array}$ \\
\hline $\begin{array}{l}\text { Contrôleur de gestion } \\
\text { «filiale» (répondant 3) }\end{array}$ & Homme & 3 & 3 & Sage, SAP & $\begin{array}{c}\text { Grande- } \\
\text { Bretagne }\end{array}$ & 1 heure \\
\hline $\begin{array}{l}\text { Contrôleur de gestion } \\
\text { «filiale (répondant 11) }\end{array}$ & Femme & 5 & 3 & non & $\begin{array}{c}\text { Paris - } \\
\text { France }\end{array}$ & 1 heure \\
\hline $\begin{array}{l}\text { Contrôleur de gestion } \\
\text { «activités (répondant 22) }\end{array}$ & Femme & 6 & 5 & Sage & $\begin{array}{c}\text { Lyon - } \\
\text { France }\end{array}$ & 1 heure \\
\hline $\begin{array}{l}\text { Contrôleur de gestion } \\
\text { «activités » (répondant 27) }\end{array}$ & Homme & 6 & 4 & Sage, & $\begin{array}{c}\text { Lyon - } \\
\text { France }\end{array}$ & $\begin{array}{c}45 \\
\text { minutes }\end{array}$ \\
\hline $\begin{array}{l}\text { Contrôleur de gestion } \\
\text { «activités 》 (répondant 28) }\end{array}$ & Homme & 4 & 4 & Sage & $\begin{array}{l}\text { Nord - } \\
\text { France }\end{array}$ & $\begin{array}{c}65 \\
\text { minutes }\end{array}$ \\
\hline
\end{tabular}

Les entretiens ont été enregistrés, retranscrits et analysés en utilisant la technique de l'analyse de contenu thématique (Wacheux, 1996 ; Bardin, 2001). Les résultats de notre analyse ont été soumis à un processus de validité interne et externe des données, comme recommandé par Wacheux (1996). 
Nous avons dans un premier temps résumé les résultats de nos entretiens et, après une première phase d'analyse, demandé aux interviewés de valider les conclusions obtenues. Le pluralisme des techniques de recherche et d'analyse (Eisenhardt, 1989 ; Woodside, 2010) a permis le recueil d'un matériau relativement riche et de combiner les analyses de données statistiques et de type qualitatif afin de renforcer la validité externe.

\section{Une évolution partielle vers le profil « demain » conditionnée à la mise en place d'un outil de BI et à la volonté de la direction générale}

Les résultats de l'enquête par questionnaire montrent un profil d'activités dans l'entreprise Alpha, proche du profil « demain » de Besson pour les volets «participer au reporting » et « gérer le système d'information de gestion » (3.1). Selon les entretiens, l'évolution sur les deux autres volets semble conditionnée à la mise en place d'un outil de BI et à la volonté de la direction d'associer le contrôleur de gestion à la planification et au pilotage (3.2).

\subsection{Une évolution partielle vers le profil d'activités « demain »}

L'analyse des résultats de l'enquête par questionnaire (tableau 1) a permis d'élaborer un profil d'activités moyen (figure 2), que nous avons mis en parallèle avec les profils d'activités « hier » et « demain » proposés par Besson (1999).

Figure 2 : Une comparaison des profils d'activités « hier » et « demain » de Besson avec le profil d'activités des contrôleurs de gestion de l'entreprise Alpha

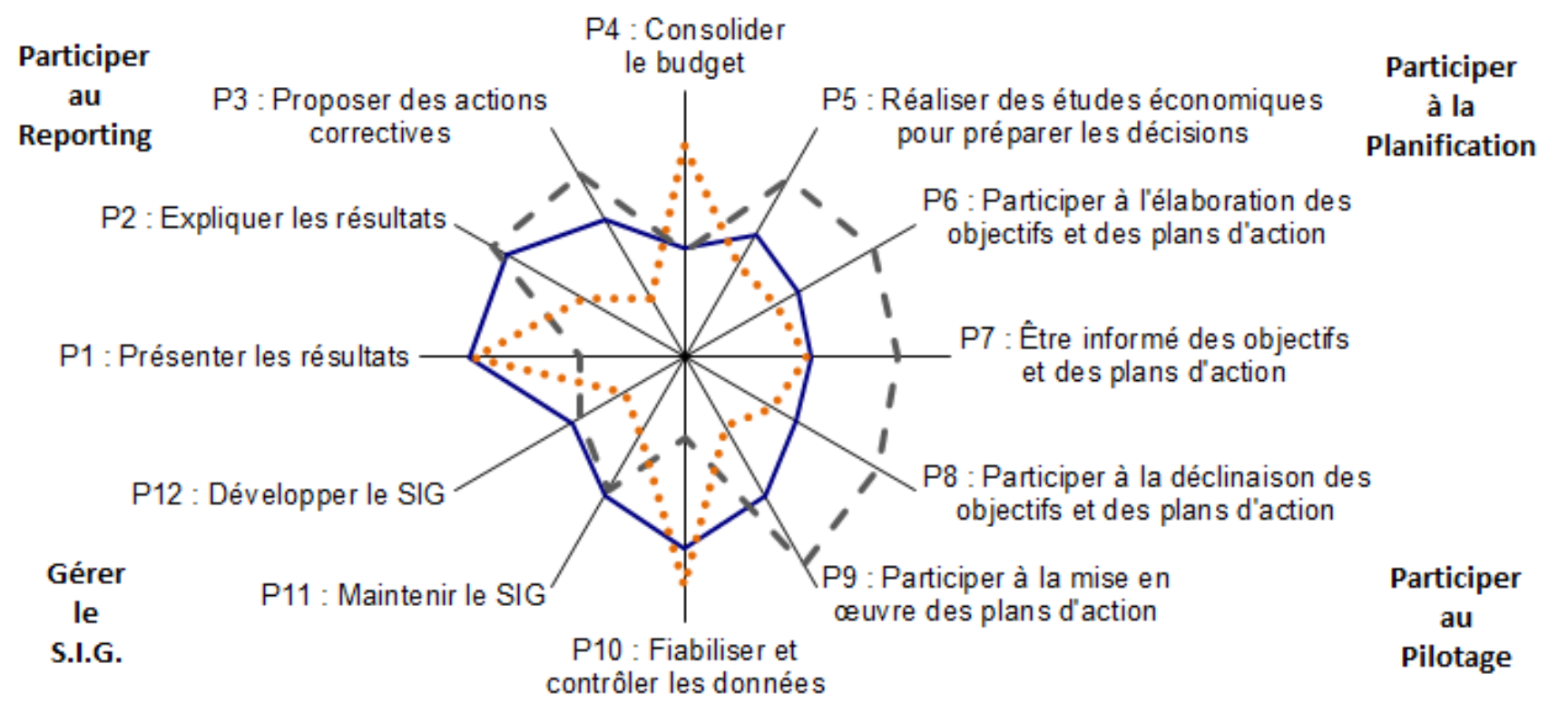

—-Profil du contrôleur de gestion dégagé de notre enquête

- Profil du contrôleur de gestion "demain" selon le modèle de Besson (1999)

..... Profil du contrôleur de gestion "hier" selon le modèle de Besson (1999)

L'analyse des quatre volets du profil d'activités du contrôleur de gestion permet de constater des points communs et des divergences avec les modèles « hier » et « demain » de Besson (1999).

\section{- Volet 1 : Participer au reporting}

L'hypothèse proposée par Besson sur ce volet est que la mise en place d'un ERP permet au contrôleur de gestion de consacrer moins de temps à la présentation des résultats (P1) et de participer plus intensément à leur explication (P2) et à la proposition de mesures correctives (P3). Les résultats obtenus confirment la plus forte implication des contrôleurs dans l'explication des résultats (P2) et, à un degré moindre que prévu, leur participation à la proposition d'actions correctives (P3). En revanche, contrairement au profil de gestion « demain » de Besson (1999), les contrôleurs de gestion 
d'Alpha consacrent encore beaucoup de temps à la présentation des résultats (P1), comme décrit dans le modèle « hier ».

\section{- Volet 2 : Participer à la planification}

L'hypothèse posée par Besson sur ce volet est que la mise en place d'un ERP permet au contrôleur de gestion de consacrer moins de temps à la consolidation des budgets (P4), et davantage à la réalisation d'études économiques pour préparer les décisions (P5), et à l'élaboration des objectifs et plans d'action (P6). Les résultats obtenus confirment que les contrôleurs consacrent moins de temps à la consolidation du budget (P4). Ils sont plus mitigés concernant leur implication dans la réalisation des études économiques pour préparer les décisions (P5) et l'élaboration des objectifs (P6). Ces résultats ne confirment que partiellement les évolutions anticipées par Besson (1999).

\section{- Volet 3 : Participer au pilotage}

L'hypothèse posée par Besson sur ce volet est que la mise en place d'un ERP permet au contrôleur de gestion d'être mieux informé des objectifs et plans d'action (P7) et de participer plus intensément à leur déclinaison (P8) et mise en œuvre (P9). Les résultats obtenus sur ces trois points sont plutôt mitigés. Les contrôleurs de gestion ne se perçoivent pas comme bien informés des objectifs et plans d'action (P7). En revanche, leur participation à la déclinaison (P8) et à la mise en œuvre des objectifs et plans d'actions (P9) est certes plus importante que dans le profil «hier», mais moins importante que dans le profil «demain » proposé par Besson.

\section{- Volet 4 : Gérer le système d'information de gestion}

L'hypothèse posée par Besson sur ce volet est que la mise en place d'un ERP permet au contrôleur de gestion de consacrer moins de temps au contrôle et à la fiabilisation des données (P10) et de s'intéresser plus intensément au maintien (P11) et au développement (P12) du système d'information de gestion. Les résultats obtenus confirment la forte implication des contrôleurs dans le maintien (P11) et le développement (P12) du système d'information de gestion, ce qui converge avec les constats opérés dans la revue de littérature. En revanche, contrairement aux attentes mises en avant par Besson (1999), ils consacrent encore beaucoup de leur temps au contrôle et à la fiabilisation des données (P10), comme le soulignent certaines recherches (Meyssonnier et Pourtier, 2006 ; Boitier, 2008 ; Chtioui et Bennani, 2009).

Au final, nos résultats montrent qu'après l'implantation de l'ERP JDE chez Alpha, le profil d'activités des contrôleurs de gestion est proche du profil «hier» pour certaines activités et proche du profil « demain » pour d'autres. La mise en place de l'ERP semble avoir impacté les activités d'analyse liées au reporting, et les activités technologiques, c'est-à-dire la conception et maintenance du système d'information de gestion. Mais les tâches traditionnelles du contrôleur de gestion, telles que la présentation des résultats, la fiabilisation et le contrôle des données sont toujours aussi importantes. Par ailleurs, l'ERP semble n' avoir qu'un impact partiel sur les activités décisionnelles et stratégiques des contrôleurs de gestion. La participation des contrôleurs de gestion d'Alpha à la prise de mesures correctives, aux études économiques pour la préparation des décisions, à l'élaboration et à la déclinaison des objectifs et des plans d'action, est plus importante que dans le profil «hier », mais moins importante que dans le profil « demain » de Besson.

Certaines convergences et certaines divergences de résultats apparaissent donc entre l'enquête de Besson (1999) et notre enquête réalisée dans la société Alpha. Plusieurs pistes sont possibles pour expliquer ces divergences. L'enquête de Besson portait tout d'abord sur les évolutions attendues par les contrôleurs de gestion, l'enquête dans la société Alpha porte sur les évolutions ex-post perçues par les contrôleurs de gestion. Ensuite, les deux groupes n'utilisent pas le même ERP (SAP pour ASF, JDE pour Alpha) et n'ont pas le même type d'activités (activité relativement standardisée - gestion des autoroutes - pour ASF, activité de services pour le groupe Alpha). Par ailleurs, l'ERP n'est pas la seule cause possible d'évolution du profil d'activités des contrôleurs de gestion. L'évolution peut être liée à des phénomènes externes : intensification du reporting avec le développement d'une société de l'audit et la financiarisation des entreprises, complexification du reporting avec l'instabilité des 
activités et périmètres de groupe. L'évolution peut aussi être liée à des phénomènes internes. Plusieurs auteurs (Fahy et Lynch, 1999 ; Booth et al., 2000 ; Granlund et Malmi, 2002 ; Rom et Rohde, 2006 ; Vakalfotis et al., 2011; Gullkvist, 2013) montrent que les systèmes SEM (Strategic Entreprise Management), ou BI (Business Intelligence) utilisés en parallèle d'un ERP, influencent le profil d'activités des contrôleurs de gestion. Afin d'approfondir et comprendre les résultats obtenus dans la première phase de l'étude et voir comment les perceptions avaient évolué, nous avons un an après l'administration des questionnaires mené six entretiens de type semi-directif.

\subsection{L'outil BI et la volonté de la direction : deux paliers pour évoluer vers le profil « demain »?}

Six entretiens ont été réalisés dans un second temps auprès du responsable du service contrôle de gestion du groupe et de 5 contrôleurs de gestion de filiales françaises et anglaises afin d'interpréter les résultats obtenus dans la première phase. Des analyses intra et inter-entretiens ont été opérées. Elles nous permettent de mieux comprendre, sur chacun des quatre volets retenus par Besson (1999), le profil d'activités des contrôleurs de gestion après la mise en place de l'ERP «JD Edwards ».

\section{- Volet 1 : Participer au reporting}

La comparaison du profil dégagé dans la phase 1 de notre étude avec celui proposé par Besson (1999) montre des résultats assez similaires sauf pour l'activité «présentation des résultats » (P1). Les contrôleurs interviewés en face-à-face dans la phase 2 de l'étude confirment qu'ils passent encore beaucoup de temps à traiter et retravailler les données de l'ERP afin de les adapter à leurs modèles de reporting. L'un d'eux explique que «l'ERP mis en place ne contient pas de structures ou d'outils de présentation de résultats très sophistiqués. Le degré d'analyse que l'ERP permet d'assurer n'est pas satisfaisant et les contrôleurs de gestion passent encore plus de temps qu'auparavant à traiter leurs résultats sur Excel ». Ce résultat est sur ce point conforme à ceux obtenus par Cross (2001) et Granlund et Malmi (2002) et à ceux de Boitier (2008, p.42-43) qui explique que : « Les contrôleurs de gestion sont cantonnés au rôle de fournisseurs de chiffres à destination du sommet stratégique... Ils assurent une traque permanente de l'information, normalement facilitée par la technologie ».

\section{- Volet 2 : Participer à la planification}

La comparaison sur ce volet du profil dégagé dans la phase 1 de notre étude avec celui du modèle de Besson (1999) confirme que, suite à la mise en place d'un ERP, le contrôleur consacre moins de temps à la consolidation du budget (P4). Un décalage apparaît en revanche au niveau des activités « réaliser des études économiques pour préparer les décisions » (P5) et « élaborer des objectifs et des plans d'actions » (P6) qui sont au-dessus du profil «hier», mais au-dessous du profil «demain ». L'analyse des entretiens met en évidence deux causes essentielles à ce décalage : l'ERP n'est ni un outil de simulation, ni un outil d'élaboration de données prévisionnelles. Un contrôleur interrogé explique que «JDE ne permet pas de faire des analyses dans le futur pour définir des objectifs » et poursuit en affirmant que "les contrôleurs de gestion réalisent quand même ces tâches mais via l'outil de Business Intelligence «MIS ${ }^{2}$ » récemment implanté chez Alpha ». Cette lacune en matière de planification et de simulation des ERP a déjà été soulignée par Brignall et Ballantine (2004) et confirmée par Vakalfotis et al. (2011) qui notent que les ERP semblent exercer une influence significative sur l'aspect transactionnel du contrôle de gestion, mais avoir un impact assez modéré au niveau prévisionnel. De même, Rom et Rohde (2006, p. 60) expliquent : «durant nos entretiens, les groupes interviewés ont confirmé la difficulté de faire de l'analyse budgétaire avec l'ERP. Les uns préfèrent utiliser des tableaux Excel pour construire leur budget et leur forecast (prévisionnel), et les autres préfèrent utiliser un outil SEM comme SAP SEM ou Cognos ». Les contrôleurs d'Alpha

\footnotetext{
${ }^{2}$ MIS est un outil de Business intelligence élaboré en 2009-2010 par le groupe Alpha lui-même en utilisant des cubes de technologie SAS. Cet outil de « reporting groupe » permet, selon le responsable du service contrôle de gestion groupe, de «faire des analyses de données à la demande et sur mesure pour tous les managers filiales, activités et groupe ». Selon lui, le groupe l'a construit afin d' "avoir une vision claire de l'activité et réaliser des mesures de performance plus sophistiquées».
} 
indiquent donc que l'ERP JDE ne leur permet pas de réaliser de simulations, ni de fournir des données prévisionnelles, mais que grâce à l'outil de Business Intelligence « MIS » récemment implanté dans l'entreprise, leurs activités sont désormais davantage orientées vers l'aide à la décision, avec une plus forte participation à la définition des objectifs, plans d'actions et propositions d'actions correctives. Il ressort ainsi de nos entretiens que, dans le groupe Alpha, l'ERP est perçu comme une simple base de données transactionnelle et que le système de BI «MIS » prend le relais pour la restitution et l'analyse des indicateurs de performance. Implémenter un ERP semble permettre d'évoluer vers une dimension plus décisionnelle lorsqu'en parallèle, des outils de BI sont mis en place pour faciliter l'exploitation des bases de données transactionnelles.

\section{- Volet 3 : Participer au pilotage}

La comparaison sur ce volet du profil dégagé par notre analyse lors de la phase 1, avec celui du modèle de Besson (1999) montre l'existence de décalages sur les trois propositions (P7, P8 et P9). Les interviews réalisées lors de la phase 2 permettent de les expliquer en partie. Premièrement, les interviewés ont tous souligné que l'ERP n'est pas un outil convivial et maniable pour réaliser des analyses de performance. Ceci rejoint les conclusions de Shields (2001, cité par Rom et Rohde, 2006), qui explique que : "L'ERP a des avantages au niveau transactionnel, mais il manque de reporting convivial et de facilités d'analyse ». Gould (2003) confirme et estime que «l'ERP est une simple base de données transactionnelle qui manque de capacité d'analyse et de mesure de performance conviviale et sophistiquée ». Deuxièmement, les contrôleurs interviewés se plaignent du fait que le développement de nouveaux tableaux de bord ou outils de mesure de performance dans l'ERP est très difficile vu la rigidité et la standardisation élevée du système. Ce constat rejoint les résultats de Davenport (1998) et de Reix (1999) qui soulignent la rigidité intrinsèque de l'outil liée à la complexité du logiciel et des méthodologies, et ceux de Booth et al. (2000) et Vakalfotis et al. (2011) qui estiment que les systèmes ERP sont des systèmes transactionnels très performants, mais avec des capacités d'analyse et d'aide à la décision limitées à cause de leur rigidité. Mais s'ils critiquent de façon assez unanime la maniabilité et les potentialités de l'ERP «JDE », les contrôleurs interrogés affirment toutefois que leur mission s'oriente désormais progressivement vers une dimension plus stratégique : de plus en plus de conseils auprès des managers, plus de participation à la mise en œuvre des plans d'action et plus de participation aux choix d'investissements et orientations stratégiques. Ils avancent que l'usage de l'ERP «JDE », combiné à l'outil de BI « MIS », a rendu possible cette évolution mais qu'il ne suffit pas à l'expliquer. Ils expliquent aussi et surtout cette évolution par la volonté récente et de plus en plus marquée de la direction générale, de les impliquer dans le processus stratégique. Le contrôleur de gestion du groupe indique en effet que "la direction souhaite désormais que les contrôleurs de gestion s'impliquent davantage dans le choix des indicateurs de performance (par activité, région, pays, filiale et business unit) et qu'ils jouent un rôle de conseiller auprès d'eux ». Il apparaît ainsi que, chez Alpha, la mise en place de l'ERP «JDE » n'a pas suffi pour faire évoluer la mission des contrôleurs de gestion du profil « hier» vers le profil «demain », avec un rôle plus stratégique. Deux autres éléments y ont contribué et l'ont rendu possible : la mise en place de l'outil de $\mathrm{BI}$ «MIS » et la volonté de la direction générale d'impliquer les contrôleurs de gestion en termes d'aide à la décision et d'exploitation des données.

\section{- Volet 4 : Gérer le système d'information de gestion}

Le profil dégagé de notre analyse dans la phase 1 et celui du modèle de Besson (1999) sont en adéquation sur les activités concernant l'implication des contrôleurs dans le maintien (P11) et le développement (P12) du système d'information de gestion. En revanche, contrairement à l'évolution anticipée par Besson (1999), notre étude fait apparaître que les contrôleurs consacrent encore beaucoup de leur temps au contrôle et à la fiabilisation des données (P10). Les contrôleurs interviewés le confirment et l'un d'entre eux explique que « le fait que le contrôleur de gestion passe encore plus de temps à contrôler et fiabiliser les données est dû à plusieurs causes. La principale concerne la mise en forme des données sorties de l'ERP. On se doute bien que le PDG du pays, le manager, ou n'importe qui, ne va pas utiliser «brut de fonderie » l'extraction directe des données de l'ERP ». Les 
contrôleurs de gestion du groupe Alpha confirment en revanche s'investir assez intensément dans la gestion du système d'information. Ils affirment que désormais la participation à la gestion du projet ERP tout au long de son cycle de vie fait partie de leurs attributions (participation aux activités d'analyse des besoins, revue de processus métiers avec les managers, conception de l'outil avec la direction informatique, paramétrages et tests ERP, mise à jour des données ou règles de gestion dans l'outil), et qu'ils sont ainsi tenus, pour survivre dans l'organisation, d'avoir cette double compétence. Ce résultat rejoint ceux de Burns et Scapens (2000), Chtioui et Bennani (2009) et Grabski et al. (2009).

\section{Discussion : L'évolution du profil du contrôleur de gestion : un processus par paliers ?}

L'étude quantitative réalisée par questionnaire dans un premier temps montre que l'évolution du profil d'activités des contrôleurs de gestion après l'implantation de l'ERP «JD Edwards » dans l'entreprise Alpha se situe principalement sur les plans analytique et technologique, ce qui confirme des résultats de recherches antérieures (Granlund et Malmi, 2002 ; Rom et Rohde, 2006). Elle montre aussi que la mise en place de l'ERP a fait émerger un contrôleur de gestion hybride capable de gérer à la fois des problématiques de contrôle de gestion «classiques » et de s'impliquer dans le maintien et le développement du système d'information de gestion. A contrario, cette première phase ne confirme que partiellement les conclusions d'autres recherches, anticipant une évolution du métier de contrôleur de gestion vers l'aide à la décision et la planification stratégique (Besson, 1999 ; Cross, 2001 ; Scapens et Jazayeri, 2003 ; Grabski et al., 2009 ; Sangster et al., 2009).

L'étude qualitative réalisée un an après montre toutefois que, petit à petit, la mission des contrôleurs de gestion évolue chez Alpha vers l'aide à la décision et la planification stratégique. Les contrôleurs interviewés expliquent que l'ERP «JD Edwards » en place dans leur entreprise n'a dans un premier temps pas été suffisant pour rendre cette évolution possible : "il reste une base de données indispensable mais ne constitue pas un outil d'aide à la décision » nous confie un contrôleur de gestion français. Ils expliquent qu'en revanche les outils de BI utilisés dans le groupe sont bien adaptés pour ce type de tâches : ils permettent la réalisation d'études et d'analyses pour l'aide à la décision et facilitent la définition des objectifs, des plans d'actions et les propositions d'actions correctives. Dans l'entreprise Alpha, l'ERP sert de bases de données aux outils de BI qui eux permettent ensuite au contrôleur de gestion de participer aux tâches d'aide à la décision, ce qui rejoint les conclusions d'autres recherches (Booth et al., 2000 ; Rom et Rohde, 2006).

Notre étude montre aussi que ces technologies (ERP et BI) sont nécessaires mais pas suffisantes pour que les missions du contrôleur de gestion prennent une dimension plus stratégique. Les contrôleurs de gestion interviewés expliquent en effet que leurs activités s'orientent, mois après mois, vers une dimension plus stratégique, que les outils technologiques constituent un support indispensable pour cela, mais que cette évolution est aussi liée à la volonté récente de la direction générale. Ils affirment en effet que leur direction affiche désormais clairement son intention de les voir s'impliquer davantage dans le choix des indicateurs de performance et dans les choix d'investissements. En d'autres termes, la direction leur demande aujourd'hui davantage qu'auparavant de participer aux orientations stratégiques et de jouer un rôle de conseiller.

Ce processus d'impact de l'ERP, des outils de BI et de la volonté de la direction générale, sur le profil d'activités du contrôleur de gestion est résumé dans la figure 3 ci-dessous : 
Figure 3 : Une relation par paliers entre ERP, outils de BI, volonté de la direction générale, et profil d'activités du contrôleur de gestion

\begin{tabular}{|c|c|}
\hline Paliers & Impact sur le profil d'activités du contrôleur de gestion \\
\hline $\begin{array}{l}\text { Palier 1: } \\
\text { Mise en place } \\
\text { de l'ERP } \\
(2007)\end{array}$ & $\begin{array}{l}\text { Pas d'impact constaté sur : } \\
\text { - Le temps consacré à la présentation des résultats (P1) } \\
\text { - } \text { Le temps consacré au contrôle et à la fiabilisation des données (P10) } \\
\text { Nouveau rôle du contrôleur de gestion (rôle hybride) sur les plans : } \\
\text { Analytique : }\left\{\begin{array}{l}\bullet \text { Moins de temps consacré à la consolidation des budgets (P4) } \\
\bullet \text { Plus de temps consacré à l'explication des résultats (P2) }\end{array}\right. \\
\text { Technologique : } \begin{array}{c}\text { ET } \\
\bullet \begin{array}{l}\text { Plus de maintenance du système d'information de gestion }(\mathrm{P} 11) \\
\text { développement du système d'information de gestion (P12) }\end{array}\end{array}\end{array}$ \\
\hline$\sqrt{b}$ & $\mathfrak{l}$ \\
\hline $\begin{array}{l}\text { Palier } 2 \text { : } \\
\text { Introduction } \\
\text { d'outils de } \\
\text { Business } \\
\text { Intelligence (BI) } \\
(2009-2010)\end{array}$ & $\begin{array}{l}\text { Une activité orientée davantage vers l'aide à la décision : } \\
\text { Décisionnel : } \begin{cases}\bullet & \text { Plus d'études économiques pour préparer les décisions (P5) } \\
\bullet & \text { Plus de participation à la définition des objectifs et plans d'actions } \\
\text { (P6) } & \text { Plus de propositions de mesures correctives (P3) }\end{cases} \end{array}$ \\
\hline$\sqrt{ }$ & $\sqrt{l}$ \\
\hline $\begin{array}{l}\text { Palier } 3 \text { : } \\
\text { Volonté de la } \\
\text { direction } \\
(2010-2011)\end{array}$ & $\begin{array}{l}\text { Participation aux décisions stratégiques : } \\
\text { Stratégique : } \begin{cases}\bullet & \text { Plus d'informations reçues des managers sur les objectifs et les } \\
\text { plans d'actions (P7) } \\
\bullet & \text { Plus de participation à la déclinaison des plans d'actions (P8) } \\
\bullet & \text { Plus de participation à la mise en œuvre des plans d'actions (P9) }\end{cases} \end{array}$ \\
\hline
\end{tabular}

Notre étude met en évidence une relation par paliers entre ERP, outils de business intelligence, volonté de la direction et le profil d'activités du contrôleur de gestion. Elle montre que l'implantation d'un ERP en 2007 (palier 1) impacte leurs mission sur les plans analytique (moins de temps consacré à la consolidation des budgets et plus à l'explication des résultats) et technologique (plus de maintenance du système d'information et de participation aux projets d'amélioration et de développement du système d'information). Elle montre ensuite que l'évolution de leurs missions vers une dimension plus décisionnelle est liée aux outils de BI mis en place en 2010 pour faciliter les analyses (un ERP couplé à un outil de BI) (palier 2). Les contrôleurs interrogés indiquent que l'ERP sert d'entrepôt de données aux analyses menées avec les outils de BI. Ces deux outils semblent complémentaires : l'ERP pour les bases de données transactionnelles, les outils de BI pour réaliser les analyses souhaitées. Elle montre enfin que l'évolution de leurs missions vers une dimension plus stratégique est liée à la volonté récente (2010-2011) de la direction (palier 3).

Au-delà du modèle de Besson (1999), cette recherche montre que l'ERP ne contribuerait à faire évoluer le métier de contrôleur de gestion vers une fonction de «partenaire » (Lambert et Sponem, 2009), ou de partenaire influent «intégré » (Bollecker, 2011) qu'à une double condition : existence d'outils de BI et volonté de la direction. Comme le soulignent Lambert et Sponem (2009), le modèle 
«business partner » fascine, mais il est peu présent dans les entreprises. Notre recherche suggère certains facteurs explicatifs à la faible prégnance du modèle «business partner » : un défaut d'outils de BI, une absence de volonté de la direction, et paradoxalement, un rôle de contrôleur de gestion évoluant avec les ERP vers les activités de maintenance du système d'information de gestion, au risque de négliger le rôle de partenaire auprès des opérationnels. Cependant, selon les entretiens, ce risque est modéré lorsque l'ERP est associé à un outil de BI. Il est alors plus facile pour le contrôleur de gestion d'assurer un rôle de partenaire et de réaliser les opérations de reporting auprès des opérationnels demandeurs.

\section{Conclusion}

Notre recherche contribue à répondre à la question posée par Besson (1999) : « le modèle du contrôleur de gestion : mythe ou réalité ? ». Les résultats montrent qu'après la mise en place d'un ERP, les tâches dites ingrates de fiabilisation et de consolidation des données restent très chronophages. Il apparait ensuite que les contrôleurs de gestion sont plus impliqués dans les activités de conception et de maintenance des systèmes d'information de gestion. L'évolution vers des activités dites nobles de support au pilotage et processus décisionnels, suppose une double condition : la mise en place d'outils de business intelligence et une volonté forte de la direction.

Comme l'ont souligné Scapens et Jazayeri en 2003, l'ERP n'est donc pas le seul levier d'évolution de la fonction «contrôle de gestion ». Ce résultat invite à engager des recherches sur l'étude de la convergence entre ERP, Business Intelligence et volonté de la direction générale, convergence quelque peu explorée dans la littérature anglo-saxonne (Zack et al, 2008) mais très peu dans la recherche francophone (El Amrani et St-Léger, 2013).

Ce travail comporte plusieurs limites qui pourront être levées par des recherches futures : une étude dans un seul groupe utilisant le progiciel JD Edwards, un nombre limité de questionnaires exploitables (30) et d'entretiens (6), et des limites liées à la mesure du temps consacré aux différentes tâches par les contrôleurs de gestion. La perception du temps passé pour chaque activité est relative, et de façon plus ou moins consciente, chacun tend peut-être à surévaluer le temps consacré aux tâches plus fastidieuses telles que la fiabilisation ou le contrôle des données. Cette recherche a pour objectif de repérer l'évolution des activités du contrôleur de gestion selon le référentiel de Besson (1999). Pour confirmer ces résultats et lever les limites liées à la perception du temps, il aurait été intéressant de recueillir le point de vue des opérationnels même si cela aurait soulevé d'autres difficultés méthodologiques car les opérationnels n'ont probablement pas une aussi forte connaissance des activités réelles des contrôleurs de gestion, ni de leur évolution.

Malgré ces limites, cette recherche permet de mettre en évidence une relation par paliers entre l'ERP, les outils de business intelligence et la volonté de la direction, relation à tester empiriquement à travers de futures recherches. Ce résultat pourrait contribuer à expliquer les causes génériques de divergence de résultats dans les recherches portant sur l'impact de l'implantation d'un ERP sur le profil d'activités des contrôleurs de gestion, mais aussi d'anticiper l'évolution des activités du contrôleur de gestion suite à l'implémentation d'un ERP. Si certains auteurs (Jourdan et al., 2008 ; Vakalfotis et al., 2011 ; El Amrani et St Léger, 2013) invitent à explorer les liens entre ERP et outils de Business Intelligence, notre travail préconise d'intégrer aussi l'attitude de la direction pour comprendre l'évolution du profil d'activités du contrôleur de gestion suite à la mise en place d'un ERP.

\section{Bibliographie}

AZAN, W., BOLLECKER, M. (2011), « Management control competencies and ERP: An empirical analysis in France. » Journal of Modelling in Management, vol 6, n², p.178-199.

BARDIN, L. (2001). L'Analyse de Contenu, Paris, PUF.

BESSON, P. (1999), «Les ERP à l'épreuve de l'organisation », Système d'Information et Management, vol. 4 , n० 4 , p. $21-51$.

BOITIER, M. (2008), «L'influence des systèmes de gestion intégrés sur l'intégration des systèmes de contrôle de gestion », Comptabilité, Contrôle, Audit, vol.14, n¹, p. 33-48.

BOLLECKER M. (2011) «La socialisation des contrôleurs de gestion, une illusion ? », Revue Gestion 2000, $\mathrm{n}^{\circ}$ 5/11. Pp. 101-122

BOOTH, P., MATOLCSY, Z., WIEDER, B. (2000), « The impacts of enterprise resource planning systems on accounting practice-the Australian experience », Australian Accounting Review, vol.10, n³, p.4-18. 
BRIGNALL, T.J., BALLANTINE. J. (2004), « Strategic Enterprise Management Systems: new directions for research», Management Accounting Research, vol. 15, n², p. 225-240.

BURNS, J., SCAPENS R.W. (2000), « Conceptualizing management accounting change: an institutional framework », Management Accounting Research, vol. 11, n¹, p. 3-25.

CAGLIO, A. (2003), « Enterprise Resource Planning Systems and Accountants: towards hybridization? », European Accounting Review, vol. 12, ${ }^{\circ} 1$, p. 123-153.

CHTIOUI, T., BENNANI, A. (2009), «Le contrôleur de gestion et les progiciels de gestion intégrée : nouvelles perspectives ou nouvelles frontières», IBIMA Business Review, vol. 4, p.8-15.

COAT, F., FAVIER, M. (1999), « Passage à l'ERP et refonte du système d'information : le cas des ASF », Systèmes d'Information et Management, vol. 4, n 4 , p.107-128.

CROSS, M. (2001), «Decision Support Systems », CMA Management, vol 75, n 9, p. 48-49.

DAVENPORT, T.H. (1998), « Putting the Enterprise into the Enterprise System », Harvard Business Review, vol 76, n 4 , p. 121-131.

EISENHARDT, K. M. (1989), "Building theories from case study research". Academy of Management Review, vol $14, \mathrm{n}^{\circ} 4$, p. $532-550$.

EL AMRANI R., ST LEGER G. (2013), «Etats des lieux de la recherche ERP francophone », Systèmes d'Information et Management, vol. 18, $\mathrm{n}^{\circ} 2$, p. 111-160.

FAHY, M. J., LYNCH, R. (1999), «Enterprise resource planning (ERP) systems and strategic management accounting », Paper presented at the EAA 22nd Annual Congress, Bordeaux, May.

GOULD, S. (2003), Improving Decision Making in Your Organization: The CIMA Strategic Enterprise Management (SEM) Initiative", CIMA, London.

GRABSKI, S.V., LEECH, S.A., SANGSTER, A.J., (2009), " "Management accountants; a profession dramatically changed by ERP systems", CIMA Research Executive Summary Series, vol.4, p 1-9

GRABSKI, S.V., LEECH, S.A., SCHMIDT, P.J., (2011), "A review of ERP research: A future agenda for accounting information systems", Journal of Information Systems, vol25, n¹, p37-78.

GRANLUND, M., MALMI, T. (2002), « Moderate impact of ERPS on management accounting: a lag or permanent outcome », Management Accounting Research, vol.13, p.299-321.

GULLKVIST, B.M. (2013), "Drivers of Change in Management Accounting Practices in an ERP Environment”, International Journal of Economic Sciences and Applied Research, vol.6, n² 2 , p.149-174.

JOURDAN, Z., RAINER R.K., MARSHALL T.E. (2008), «Business Intelligence: An Analysis of the Literature », Information Systems Management, vol. 25, n², p. 121-131.

LAMBERT C., SPONEM S. (2009), "La fonction contrôle de gestion : proposition d'une typologie », Comptabilité Contrôle Audit, vol. 15, $\mathrm{n}^{\circ}$ 2, p. 113-144.

MEYSSONNIER, F., POURTIER, F. (2006), «Les ERP changent-ils le contrôle de gestion ? , Comptabilité Contrôle Audit, vol. 1, p. 45-64.

O'MAHONY, A., DORAN, J. (2008), « The changing role of management accountants; Evidence from the implementation of ERP Systems in large organisations », International journal of business management, vol. $3, n^{\circ} 8$, p. $109-115$.

REIX, R. (1999), «Les technologies de l'information, facteur de flexibilité ? », Revue Française de Gestion, $\mathrm{n}^{\circ} 123, \mathrm{p} .111-118$.

REIX, R., FALLERY, B., KALIKA, M., ROWE, F., (2011), Systèmes d'information et management des organisations, Vuibert, 6 ème Ed.

ROM, A., ROHDE, C. (2006), «Enterprise Resource Planning (ERP) Systems, Strategic Enterprise Management Systems and Management Accounting: a Danish Study», Journal of Enterprise Information Management, vol. 19, $\mathrm{n}^{\circ} 1$, p. 50-66.

ROWE, F. (1999), "Cohérence, intégration informationnelle et changement: esquisse d'un programme de recherche à partir des progiciels Intégrés de gestion », Systèmes d'Information et Management, V4, p.3-20

SANGSTER, A., LEECH, S.A, GRABSKI, S. (2009), "ERP implementations and their impact upon management accountants", Journal of Information Systems and Technology Management, vol.6, n2, p125-142 SCAPENS, R., JAZAYERI, M., SCAPENS, J. (1998), «SAP: Integrated Information Systems and The Implications For Management Accountants », Management Accounting, Sept, p. 46-48.

SCAPENS, R.W., M. JAZAYERI (2003), «ERP systems and management accounting change: opportunities or impacts? A research note », European Accounting Review, Vo12, n¹, p201-233.

VAKALFOTIS, N., BALLANTINE, J., WALL, A. (2011), «A literature review on the impact of Enterprise Systems on management accounting », Proceedings of the 8th International Conference on Enterprise Systems, Accounting and Logistics (ICESAL), Greece, p. 79-105.

WACHEUX, F. (1996), Méthodes qualitatives et recherche en gestion, Economica, 290 pages.

WOODSIDE, A. G. (2010), Case study research: Theory, methods and practice, Emerald Group Publishings. YIN, R.K., (2008), Case Study Research: Design and Methods, Thousand Oaks, Sage, 181 pages.

ZACK J., RAINER R.K., MARSHALL T.E. (2008), "Business Intelligence: An Analysis of the Literature", Information Systems Management, 25, p. 121-131. 